IDDF2018-ABS-0056 LONG TERM OUTCOMES OF INITIAL INFLIXIMAB THERAPY FOR INFLAMMATORY POUCH PATHOLOGY: A MULTI-CENTRE RETROSPECTIVE STUDY

'Jonathan Segal*', 'Lawrence Penez, ${ }^{2}$ Soad Elkady, ${ }^{1}$ Guy Worley, ${ }^{3}$ Simon McLaughlin, ${ }^{4}$ Benjamin Mullish, ${ }^{5}$ Mohammed Quraishi, ${ }^{6}$ Nik Ding, ${ }^{6}$ Tamara Glyn, ${ }^{7}$ Kesavan Kandiah, ${ }^{8}$ Mark Samaan, ${ }^{8}$ Peter Irving, ${ }^{1}$ Omar Faiz, ${ }^{1}$ Susan Clark, ${ }^{1}$ Ailsa Hart. ${ }^{1}$ St Mark's Hospital, Harrow, UK; ${ }^{2}$ Department of Gastroenterology, Faculty of Medicine, University of Alexandria, Egypt; ${ }^{3}$ Department of Gastroenterology, The Royal Bournemouth and Christchurch Hospitals, Bournemouth, UK; ${ }^{4}$ Division of Digestive Diseases, St Mary's Hospital Campus, Imperial College London, UK; ${ }^{5}$ Institute of Translational Medicine, University of Birmingham, Department of Gastroenterology, University Hospital,Birmingham, UK; ${ }^{6}$ Department of Gastroenterology, St Vincent's Hospital Melbourne, Australia; ${ }^{7}$ Department of Gastroenterology, Queen Alexandra Hospital, Portsmouth, UK; ${ }^{8}$ Department of Gastroenterology, Guy's and St Thomas' NHS Foundation Trust, London, UK

\subsection{6/gutjnl-2018-IDDFabstracts.89}

Background Restorative proctocolectomy is considered the procedure of choice in patients with UC refractory to medical therapy. Inflammation of the pouch is a common complication and in some cases fails to respond to antibiotics, the mainstay of treatment. In such cases, corticosteroid, immunomodulatory or biologic treatments are an option. However, our understanding of the effectiveness of IFX for both chronic pouchitis and Crohn's-like inflammation are based on small studies.

Methods This was an observational, retrospective, multi-centre study to assess the effectiveness IFX for inflammatory disorders related to the pouch. The primary outcome was the development IFX failure defined by primary non-response or secondary loss of response to IFX.

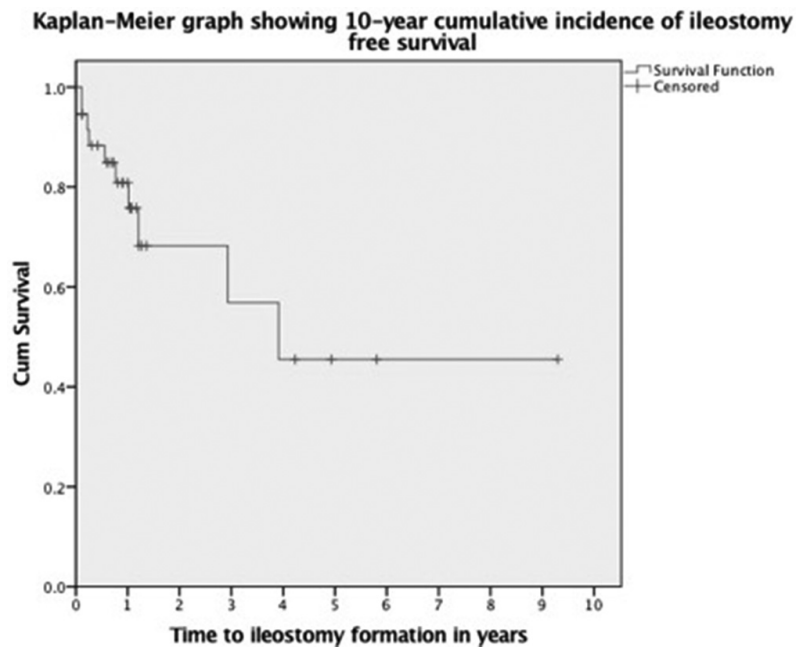

Abstract IDDF2018-ABS-0056 Figure 1 Kaplan-Meier graph showing 10-year cumulative incidence of ileostomy free survival

Results 38 patients were included. 20/38 (53\%) who were initiated on IFX for inflammatory disorders of the pouch had IFX failure, 4/38 (11\%) had primary non-response, and 16/38 $(42 \%)$ had secondary loss of response with a median followup of 265 days (range 82-2119). Of those that had IFX failure $10 / 20(50 \%)$ avoided an ileostomy by switching to an alternative biologic. In total, 28/38 (74\%) avoided an ileostomy, of these, $17 / 38(45 \%)$ continued on their IFX after a median follow-up of 311 days (42-3968), 5/38 (13\%) were changed to Adalimumab after a median follow-up of 498 days
(1-1544), 4/38 (11\%) were changed to Vedolizumab after a median follow-up of 569 (251-1138), 1 achieved histological remission and stopped all treatments at 251 days and 1 was maintained on methotrexate and multiple antibiotics after 3968 days.

Conclusions After initial IFX therapy over half will fail first line IFX, of those that fail half can avoid an ileostomy by switching to an alternative biologic. Patients should be counselled about a high incidence of failure and alternatives should be considered.

\section{IDDF2018-ABS-0057 BIOFEEDBACK IN PATIENTS WITH ILEOANAL POUCH DYSFUNCTION: A SPECIALIST CENTRE EXPERIENCE}

Jonathan Segal*, Heyson Chan, Brigitte Collins, Omar Faiz, Susan Clark, Ailsa Hart. St Mark's Hospital Harrow, UK

\subsection{6/gutjnl-2018-IDDFabstracts.90}

Background Restorative proctocolectomy is performed in patients with UC refractory to medical therapy, UC related neoplasia, and in some patients with familial adenomatous polyposis. Incontinence can occur in up to $12 \%-31 \%$ of patients with an ileoanal pouch. Incontinence and evacuatory disorders associated with the ileoanal pouch can be particularly problematic and difficult to treat using conventional therapies.

Biofeedback therapy is a behavioural treatment which is non-invasive and offers a non-surgical approach as an alternative or adjunct for patients with functional bowel disorders. The theoretical basis for biofeedback is 'learning through reinforcement' or 'operant conditioning'.

Methods This was a retrospective single centre study. All patients attending for biofeedback at our institution between Jan 2012 and Oct 2017 were identified to include all those that attended for a pouch related problems. We recorded patient reported subjective improvements following biofeedback. The validated International Consultation on Incontinence Questionnaire was used to assess improvement in incontinent symptoms, and the evacuatory disorder questionnaire was used to assess improvement in evacuatory disorders.

Results $n=26$. Based on patients ${ }^{6}$ feedback at next clinical encounter following biofeedback, nine reported much improvement, 11 reported some improvement and six reported no improvement. In the group treated for incontinence, quality of life improved significantly from a median pre-treatment score of 80 to a post-treatment score of $41(p=0.01)$ (table 1). Biofeedback reduced pain, bloating, straining and laxative use in patients with evacuatory disorders.

Abstract IDDF2018-ABS-0057 Table 1 Incontinence disorder using objective scoring system $(n=5)$ ICIQb Scores

\begin{tabular}{lccc}
\hline Domain & $\begin{array}{c}\text { Pre-biofeedback score (median, } \\
\text { range) }\end{array}$ & $\begin{array}{c}\text { Post biofeedback score (median, } \\
\text { range) }\end{array}$ & $\begin{array}{c}P \\
\text { value }\end{array}$ \\
\hline $\begin{array}{l}\text { Bowel } \\
\text { pattern }\end{array}$ & $62(49-62)$ & $46(39-62)$ & 0.12 \\
Bowel & $82(33-102)$ & $53(11-76)$ & 0.21 \\
control & & & \\
Non-scored & $22(17-35)$ & $29(12-29)$ & 0.35 \\
Quality of & $80(62-98)$ & $41(30-55)$ & 0.01 \\
life & & & \\
\hline
\end{tabular}


Conclusions Biofeedback is associated with significant improvement in the quality of life as well as possible improvements in symptoms related to both incontinence and evacuatory disorders. It is probably an underused service. Further larger prospective studies are required to assess the efficacy of biofeedback in pouch related dysfunction.

\section{IDDF2018-ABS-0059 IMMUNOGENICITY OF QUADRIVALENT INFLUENZA VACCINE FOR PATIENTS WITH INFLAMMATORY BOWEL DISEASE}

Yasuhisa Sakata*, Shimpei Shirai, Megumi Hara, Nanae Tsuruoka, Ryo Shimoda, Kazuma Fujimoto. Saga Medical School, Japan

\subsection{6/gutjnl-2018-IDDFabstracts.91}

Background No reports have described the immunogenicity and boosting effect of the quadrivalent influenza vaccine in adults-patients with inflammatory bowel disease (IBD).

Methods Adults with Crohn's disease or ulcerative colitis were randomly assigned to a single vaccination group or booster group, and a quadrivalent influenza vaccine was administered subcutaneously. Serum samples were collected before vaccination, 4 weeks after vaccination and after the influenza season in the single vaccination group and before vaccination, 4 weeks after the first vaccination, 4 weeks after the second vaccination, and after the influenza season in the booster group. We measured hemagglutination inhibition antibody titer and calculated the geometric mean titer ratio, seroprotection rate, and seroconversion rate.

Results Totally, 132 patients were enrolled. Twenty-two patients received immunomodulatory monotherapy and 16 received anti-TNF- $\alpha$ single-agent therapy. Fifteen patients received combination therapy comprising an immunosuppressant and anti-TNF- $\alpha$ agent. Each vaccine strain showed immunogenicity satisfying the European Medicines Agency criteria with a single inoculation. The booster influenza vaccination did not induce an additional response. In patients administered infliximab, the seroprotection rate and seroconversion rate tended to be lower in patients who maintained blood concentrations (seroprotection rate: H1N1: OR, $0.37 \quad(95 \%$ CI, 0.11-1.21); H3N2: 0.22 (0.07-0.68); seroconversion rate: H1N1: 0.23 (0.06-0.91); H3N2: 0.19 (0.06-0.56)).

Conclusions Although single-dose quadrivalent influenza vaccine showed sufficient immunogenicity in the patients with inflammatory bowel disease, the immunogenicity of patients receiving infliximab therapy was low.

\section{IDDF2018-ABS-0060 A STUDY ON THE STATUS OF PROTON PUMP INHIBITOR PRESCRIBED FOR HOSPITAL INPATIENTS IN JAPAN}

Yasuhisa Sakata*, Nanae Tsuruoka, Ryo Shimoda, Daisuke Yamaguchi, Kazuma Fujimoto. Saga Medical School, Japan

\subsection{6/gutjnl-2018-IDDFabstracts.92}

Background In Japan, the amount of usage of proton pump inhibitors (PPIs) has been increasing as the indications extended to maintenance treatment of reflux esophagitis and prevention of ulcer recurrence in patients receiving non-steroidal anti-inflammatory agents (NSAIDs) and low-dose aspirins, in addition to the treatment of peptic ulcer. This study aimed to clarify the status of use of PPIs in hospitalised patients with Diagnosis Procedure Combination (DPC) data.

Methods A retrospective observational study was carried out. We analysed the PPI prescription data for past 8 years (2009_ 2016) using the DPC survey data of approximately ten thousand patients a year in Saga university hospital. The primary objective is to investigate the trend of a number of hospitalised patients who were prescribed PPI for the past 8 years. The secondary objective is to investigate the changes in the characteristics of patients (age, indication, concomitant medication, and clinical department that follows a patient). Lists of proton pump inhibitors subjected to the survey are omeprazole, esomeprazole, lansoprazole, rabeprazole and vonoprazan.

Results We identified 11009 patients using PPIs throughout the study period. Prevalence of PPI use was higher among male than female, approximately 1.3 times. The average age of PPI users was 67.8 years. The number of PPI users increased 1.5 times during the study period, from 1071 (10.5\% of inpatients) in 2009 to 1581 (12.3\% of inpatients) in 2016. Lansoprazole was the most commonly used agent $(30.7 \%-50.5 \%)$ during the study period, followed by rabeprazole (29.3\%-48.6\%) and esomeprazole. A newer agent, vonoprazan exhibited substantial growth in prescription rates $(2.4 \%$ in 2015 and $13.8 \%$ in 2016). In most of the clinical departments, especially emergency and critical care medicine, neurology, cardiovascular surgery and nephrology, the number of patients prescribed PPIs was up to 3 times higher in the second half of the period (2013-2016) than in the first half of the period (2009-2012). Patients received PPIs concomitantly with NSAIDs and antithrombotic agents has been increased.

Conclusions The use of PPIs, as well as the approved indications for PPIs, has increased substantially in hospitalised patients. The prevalence of PPI prescription by doctors other than gastroenterologists also increased.

\section{IDDF2018-ABS-0061 RISK OF TUBERCULOSIS IN INFLAMMATORY BOWEL DISEASE AND OTHER IMMUNE- MEDIATED DISEASES ON BIOLOGICAL THERAPY: A POPULATION-BASED STUDY IN HONG KONG}

${ }^{1}$ Xing Wang*, ${ }^{2}$ Sunny Wong, ${ }^{2}$ Xian-Song Wang, ${ }^{2}$ Whitney Tang, ${ }^{1}$ Bin Wu, ${ }^{2}$ Justin Wu, ${ }^{2}$ Francis Chan, ${ }^{2}$ Joseph Sung, ${ }^{2}$ Siew Ng. ${ }^{1}$ Department of Gastroenterology, The Third Affiliated Hospital of Sun Yat-sen University, China; ${ }^{2}$ Department of Medicine and Therapeutics, Institute of Digestive Diseases, The Chinese University of Hong Kong, Hong Kong

\subsection{6/gutjnl-2018-IDDFabstracts.93}

Background Biological therapies are increasingly used to treat inflammatory bowel disease (IBD) and other immune-mediated diseases. However, real-world epidemiological data on the risk of tuberculosis (TB) in these patients are scarce. We investigated the incidence of $\mathrm{TB}$ in patients with immune-mediated diseases in a population-based setting and stratified the risk of TB among different biologics.

Methods Data on patient demographics, disease diagnosis, types of biologics and TB infections were collected from a territory-wide computerised database of patient records managed by the Hong Kong Hospital Authority. We calculated the incidence rates (IRs) of TB infections in subjects treated with different biologics between 2006 and 2015, and reported 